\title{
UNA VISIÓN DE LAS HUMANIDADES DIGITALES A TRAVÉS DE SUS CENTROS
}

\section{Esteban Romero-Frías y Salvador Del-Barrio-García}
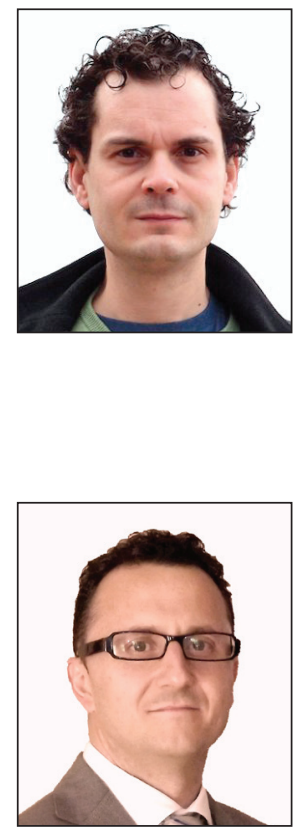

Esteban Romero-Frías es profesor de la Universidad de Granada. Sus trabajos de investigación se han centrado principalmente en la aplicación de técnicas webmétricas al campo de la empresa y la política, así como en el empleo de tecnologías digitales en educación. Trabaja en temas de culturas digitales, digital scholarship y ciencias sociales y humanidades digitales. Coordina GrinUGR, colaboratorio sobre culturas digitales en ciencias sociales y humanidades.

http://estebanromero.com

http://grinugr.org

http://orcid.org/0000-0003-2205-3560

Universidad de Granada, Facultad de Ciencias Económicas y Empresariales Departamento de Economía Financiera y Contabilidad Campus Universitario de Cartuja. 18071 Granada, España erf@ugr.es

Salvador Del-Barrio-García es profesor titular de comercialización e investigación de mercados en la Universidad de Granada y coordinador del máster oficial en marketing y comportamiento del consumidor de la misma universidad. Sus líneas de investigación se han centrado en el estudio de la eficacia publicitaria, la comunicación integrada de marketing, el comportamiento del consumidor desde una perspectiva cross-cultural y el neuromarketing. Miembro de GrinUGR, trabaja en temas de ciencias sociales y humanidades digitales.

http://marketing.ugr.es

http://orcid.org/0000-0002-6144-0240

Universidad de Granada, Facultad de Ciencias Económicas y Empresariales Departamento de Comercialización e Investigación de Mercados Campus Universitario de Cartuja. 18071 Granada, España dbarrio@ugr.es

\section{Resumen}

Las humanidades digitales se han organizado institucionalmente en la academia de formas muy diversas (centros, redes, proyectos, etc.), adaptándose según las circunstancias con el fin de ocupar un espacio propio. Se aborda la situación de las humanidades digitales a partir del análisis de sus centros, tal y como aparecen recogidos por la organización CenterNet. Se presenta una descripción global de los mismos a partir de la información de sus webs, del modo en que se definen a sí mismos y de su presencia en medios sociales. Los resultados muestran una gran heterogeneidad en las humanidades digitales, dejando en evidencia cuestiones ampliamente discutidas como los límites del campo, los problemas de inclusión y exclusión o las nuevas formas de colonialismo cultural.

\section{Palabras clave}

Humanidades digitales, Centros de humanidades digitales, Informática humanística, Digital scholarship, e-Investigación, Centros, Humanidades, Ciencias sociales.

\section{Title: A view of digital humanities through its study centers}

\section{Abstract}

Digital humanities (DH) have adopted various institutional forms in academia (centres, networks, projects, etc.). This study addresses the current state of the discipline through an analysis of DH centres, as reported by CenterNet. We analysed websites, missions, and the social media presence of the centres in order to provide an overall picture. Results show a remarkable heterogeneity in $\mathrm{DH}$ organisation. Finally, some of the most debated issues are discussed, such as the borders of the field, inclusion and exclusion issues, or a new cultural colonialism.

\section{Keywords}

Digital humanities, DH, Digital humanities centers, Humanities computing, Digital scholarship, e-Research, Centres, Humanities, Social sciences.

Romero-Frías, Esteban; Del-Barrio-García, Salvador (2014). "Una visión de las humanidades digitales a través de sus centros". El profesional de la información, septiembre-octubre, v. 23, n. 5, pp. 485-492. 


\section{Introducción}

La humanidades digitales (en adelante, DH) constituyen un campo emergente que actualmente se configura a partir de una doble tradición. En primer lugar, la corriente que se conoce entre otros nombres como humanities computing (McCarty, 2005). Ésta se remonta a los años cuarenta del siglo XX, cuando el investigador jesuita Roberto Busa inicia el proyecto de crear una concordancia en latín de las obras de Santo Tomás de Aquino (Hockey, 2000, p. 5) empleando la computación que entonces brindaba la empresa IBM. Las humanities computing (en español se conoce, entre otras formas, como informática humanística (Lucía-Megías, 2003) se centran en la aplicación de la informática a problemas propios de las humanidades con un sesgo muy importante hacia temas de análisis textual. En segundo lugar, las $\mathrm{DH}$ recogen en el área de humanidades las propuestas de digital scholarship (Weller, 2011), que van más allá de la adopción de la computación como método de investigación, y que en buena medida se centran en los cambios en las formas de comunicación y colaboración en entornos académicos a partir de la adopción de las tecnologías digitales.

Desde que en 2004 el campo se rebautizara con el nombre de $\mathrm{DH}$ a partir del libro $A$ companion to digital humanities editado por Schreibman, Siemens y Unsworth (2004) se ha ampliado con esta segunda vía. Si bien ambas genealogías comparten características e intereses comunes, es cierto que una parte de los nuevos interesados en $\mathrm{DH}$, particularmente tras la popularización y adopción en la academia de la Web 2.0 (Davidson, 2008), están interesados en el fenómeno digital en su conjunto y no tanto en la perspectiva instrumental que hasta ahora ha respondido principalmente a una tradición textual (Svensson, 2009).

Así, hoy en día las DH constituyen un concepto paraguas cuya definición (Berry, 2012; Burdick et al., 2012; Gold, 2012; Terras; Nyhan; Vanhoutte, 2013) es más difícil de concretar que nunca y cuya amplitud permite dar cobijo a

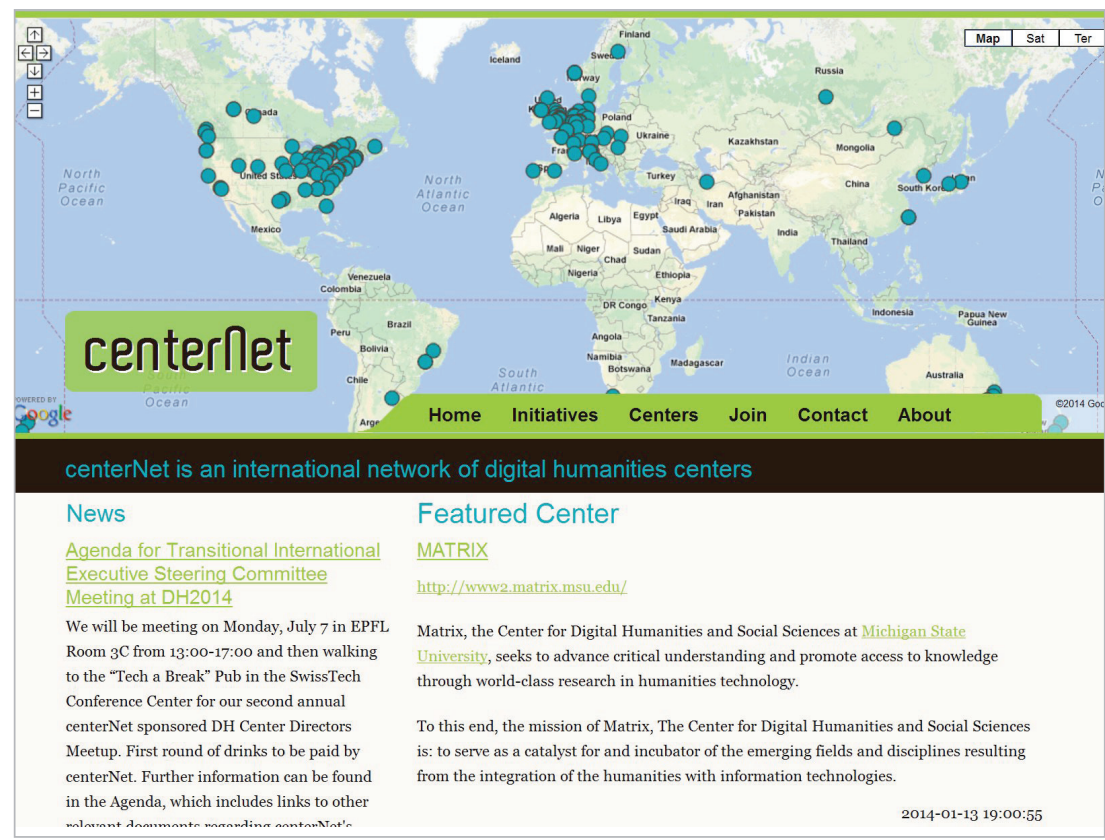

http://digitalhumanities.org/centernet investigadores, docentes y otros interesados que trabajan en los nuevos espacios generados por la intersección entre humanidades y tecnologías de la información. Se habla de las $\mathrm{DH}$ como una gran tienda (big tent) que acoge a cualquier interesado en el campo (Terras, 2011).

Como campo emergente ha ido desarrollando formas de organización particulares, si bien ha sido común la creación de centros, particularmente en universidades estadounidenses y en el Reino Unido. En cambio, los departamentos, forma tradicional de reconocimiento académico de una disciplina, son más escasos (King's College London presenta una de las excepciones más destacadas). Las asociaciones y redes han cumplido un papel muy destacado en la articulación de las diversas comunidades de humanistas digitales, así como determinados proyectos que han permitido crear grupos de trabajo estables.

El presente trabajo tiene por objetivo analizar las $\mathrm{DH}$ a partir del modo en que se organizan institucionalmente. Para ello prestaremos especial atención a la forma en que los diversos centros se definen a sí mismos, así como a la relación entre sus características y su presencia en medios sociales, forma de presencia genuina del entorno digital del que las $\mathrm{DH}$ son bandera en la academia. Ello nos servirá para examinar algunas de las expectativas y dificultades actuales del campo.

\section{Las humanidades digitales a través de sus centros}

Como avanzamos en el apartado anterior, la tipología de "centros" de DH es muy amplia. Zorich (2008, p. 7), define un centro de $\mathrm{DH}$ como "una entidad donde se utilizan los nuevos medios y tecnologías para la investigación y la enseñanza de las humanidades, con experimentación y compromiso intelectual". Svensson (2009) apunta que la institucionalización de iniciativas de humanities computing se ha producido de formas muy diversas. Generalmente estos centros ocupan una posición en la academia que suele ser transdisciplinar. Aunque en los últimos años se han creado numerosas instituciones al calor de la popularización de las $\mathrm{DH}$, buena parte de los referentes en el campo proceden de la tradición computacional que se remonta años atrás (Terras, 2006).

Entender el contexto organizacional representa aún un reto en $\mathrm{DH}$ (Siemens, 2009). Warwick (2004) exploró el contexto organizativo de centros en Estados Unidos e Inglaterra. Zorich (2008) se centró en Estados Unidos. Kirschenbaum (2010) abordó la formación del concepto de $\mathrm{DH}$ y de los centros y asociaciones que articulan el movimiento. Unsworth (2007), a propósito del informe sobre ciberinfraestructuras del American Council of Learned Societies $(A C L S)$, señala que "los centros de humanidades digitales son ciberinfraestructuras para las humanidades y las ciencias sociales; no es el único tipo, pero es uno de los más importantes". 


\section{Metodología}

\subsection{Selección de los centros de humanidades digita- les objeto de análisis}

Teniendo en mente la propia controversia sobre la naturaleza del campo, la determinación de qué es un centro de $\mathrm{DH}$ no resulta sencilla. Algunas iniciativas como Day of $\mathrm{DH}$ o Atlas de ciencias sociales y humanidades digitales emplean criterios basados en la autoidentificación por parte de los propios miembros de la comunidad de $\mathrm{DH}$.

http://dayofdh2014.matrix.msu.edu

http://atlascshd.org

Se ha tomado como población objeto de estudio el conjunto de centros recogido por CenterNet, organización que combina un sistema que podríamos denominar de autoidentificación más suscripción mediante una cuota.

Para su elección se esgrimen varias razones:

- Constituye una red internacional de centros de humanidades digitales que forma parte de la Alliance of Digital Humanities Organizations $(A D H O)$, organismo internacional de referencia.

http://adho.org

- Incluye centros con una perspectiva global, incluyendo todos los continentes y varias lenguas, a diferencia, por ejemplo, de Atlas de ciencias sociales y humanidades digitales o del listado que publica Hastac (Humanities, Arts, Science, and Technology Alliance and Collaboratory). http://www. hastac.org/content/listing-digitalhumanities-centers-and-institutes

- Se centra exclusivamente en centros, no en investigadores, proyectos u otros recursos.

- Desde un punto de vista de la visibilidad en internet, si realizamos una búsqueda en Google con los términos "digital humanities centers" el primer resultado es CenterNet.

Finalmente CenterNet ha sido tomado como referencia para comparaciones en análisis que otros investigadores han realizado. Por ejemplo, Ortega y Gutiérrez (2014) apuntan que presenta la limitación de tener un sesgo hacia lo anglosajón.

A fecha de 4 de abril de 2014 el listado de centros publicado estaba formado por 193 entidades. Algunas de las entidades que CenterNet cataloga no responden como tal a un centro o red, sino que en algunos casos se incluyen programas de estudio o proyectos (en algunos casos concluidos). Con el fin de preservar la diversidad de la comunidad de $\mathrm{DH}$ siempre que las entidades proporcionaran información sobre su naturaleza o sus misiones, se ha decidido incluirlas en el estudio, ya que ha sido en ocasiones a través de estas formas como se han desarrollado grupos o comunidades de humanistas digitales.

Tras una primera revisión, fueron descartados 37 centros por las siguientes razones:

- Información insuficiente en la web del centro para determinar su naturaleza o misión: 13 centros eliminados.

- Referencia a programas de estudios u otros servicios sin información suficiente para determinar su misión: 7 .

- Web del centro no disponible o enlace incorrecto sin po- sibilidad de determinar el adecuado a través de buscadores: 16 .

- Centros repetidos en el listado de CenterNet: 1.

\subsection{Proceso de codificación de los centros}

En primer lugar se examinaron los enlaces a sus sitios web de acuerdo con lo establecido en CenterNet. En el caso de que hubiera incorrecciones se realizó su búsqueda directamente a través de Google.

En segundo lugar se capturó la información acerca de la naturaleza y propósito de los centros, tal y como aparecía recogida en las declaraciones de "about", "mission", "vision", "who we are" o similares. La información fue siempre capturada directamente en inglés, español, francés o a través de traducciones al inglés, mediante Google Translator, en el caso de que estuviera en otro idioma.

En tercer lugar se determinó la presencia en Twitter y Facebook de los centros, bien a través de indicaciones en su propia web o a través de búsquedas directas en dichas redes o en Google. Sólo se consideró la presencia en redes cuando fuera exclusiva del centro en cuestión, no por ejemplo si correspondía a una universidad en su conjunto. En el caso de Facebook la presencia suele ser en forma de página, salvo algunas excepciones en que se emplean grupos o perfiles.

Tabla 1. Items de los mission statements empleados en el análisis de centros

\section{A. Propósito de la organización}

A1. Desarrollo de comunidades de interesados y público en general, comprometidos con las humanidades, con el fin de compartir experiencias y recursos así como crear espacios de colaboración.

A2. Crítica de las formas tradicionales de conocimiento y aprendizaje y desarrollo de proyectos innovadores.

A3. Promoción del activismo y compromiso social.

A4. Desarrollo de infraestructuras para las humanidades digitales (incluyendo herramientas, metodologías, recursos, contenidos digitales...)

A5. Fomento de la interdisciplinariedad.

B. Cómo alcanzar el propósito

B1. Proporcionando infraestructuras, incluyendo la creación de herramientas, servicios y las condiciones necesarias para el desarrollo de las humanidades digitales.

B2. Creación de espacios para el diálogo y el aprendizaje.

B3. Diseño y participación en proyectos de investigación.

B4. Proporcionando asistencia técnica a los interesados en humanidades digitales.

C. Principios que guían el propósito (traducidos directamente de Zorich, 2008, p. 15)

C1. El valor perdurable de las humanidades.

C2. Colaboración e interdisciplinariedad.

C3. Apertura (openness).

C4. Responsabilidad cívica y social.

C5. Cuestionamiento de las "vacas sagradas". 
En Twitter se registró el número de tweets publicado por cada perfil y en Facebook el número de "me gusta" o de amigos.

Finalmente, para cada centro se capturó información descriptiva incluyendo: país (en el caso de redes internacionales se adopta el país donde está su sede), los idiomas empleados, el tipo de entidad de que se trata (por ejemplo, centro como tal u otras formas), el tipo de licencia empleada en la web (con copyright o sin indicación expresa, o con licencia Creative commons), o si se hacía mención expresa a la ciencias sociales.

Esta información se recogió el 4 de mayo de 2014.

\subsection{Clasificación de los centros}

Para la codificación de las características de los centros se tomó como referencia el trabajo de Zorich (2008), que analizó los mission statements de 32 centros de humanidades digitales en los Estados Unidos clasificándolos en tres categorías:

A) Propósito de la organización (formado por 13 elementos).

B) Cómo alcanzar el propósito (10 elementos).

C) Principios que guían el propósito (5 elementos).

En nuestro caso, para hacer el análisis más operativo redujimos el número de elementos en cada categoría a partir del consenso alcanzado entre tres expertos que los agruparon de acuerdo con sus similitudes. Dado que en la categoría C sólo existían 5 elementos, se tomó directamente.

La tabla 1 muestra las 3 categorías y los elementos finalmente incluidos en cada una de ellas como instrumento de clasificación.

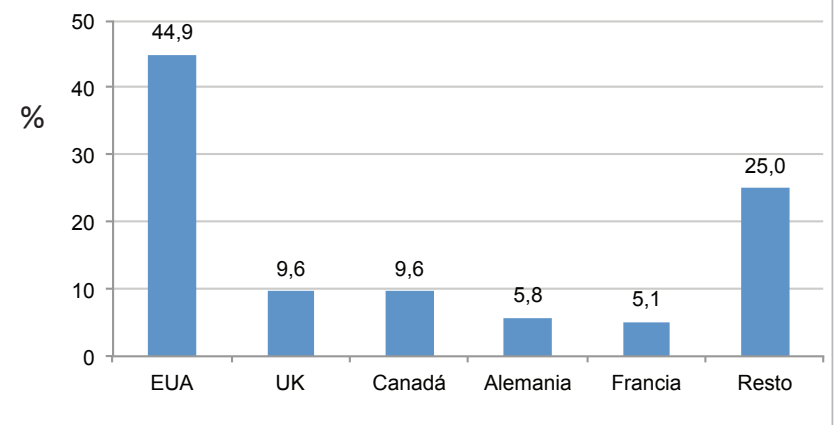

Gráfico 1. Centros de DH por país (en porcentaje)

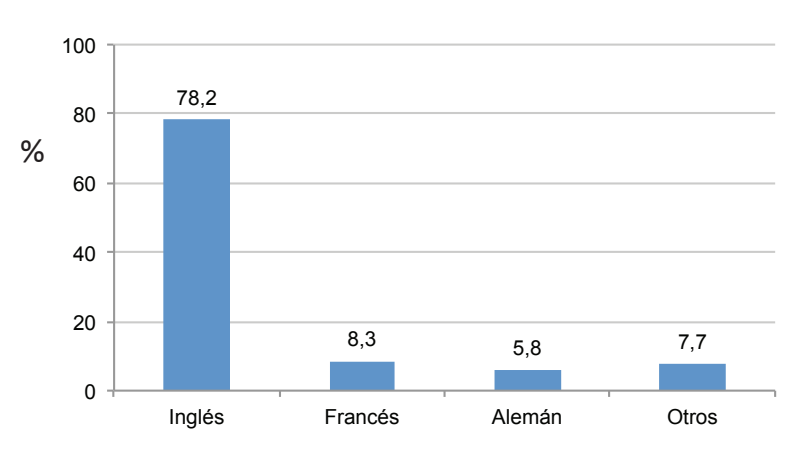

Gráfico 2. Centros de DH por idioma en el que aparece la información (en porcentaje)

\section{Resultados y discusión}

\subsection{Descripción de los centros de humanidades di- gitales}

Las figuras 1, 2 y 3 recogen los datos de los centros analizados según las variables de clasificación. Se observa el predominio del mundo anglosajón en este tipo de centros dado que 100 de los 156 centros pertenecen a Estados Unidos, Reino Unido y Canadá. Les siguen en cuarto y quinto puesto Alemania $(5,8 \%)$ y Francia $(5,1 \%)$. Estos 5 países representan el $75 \%$ de los centros de $\mathrm{DH}$ en el mundo, de acuerdo con la visión que CenterNet proporciona.

Este dominio se evidencia en el idioma en que operan los centros, ya sea como única lengua o como lengua prioritaria: inglés $(78,2 \%)$, seguido a gran distancia por el francés $(8,3 \%)$ y el alemán $(5,8 \%)$. Al margen, gran parte de las entidades que emplean otra lengua ofrecen información adicional en inglés.

Si tenemos en cuenta el tipo de entidad de que se trata, el $63,5 \%$ de las contempladas en el trabajo obedece a la figura de centro propiamente dicho [por ejemplo, Center for Information Modelling in the Humanities en Graz, Austria; Centre for Research in Arts, Social Sciences and Humanities (Crassh), en la University of Cambridge; o el HyperStudio del MIT] como unidad generalmente transdisciplinar que articula iniciativas de investigación, docencia y en ocasiones soporte técnico en las áreas vinculadas a las $\mathrm{DH}$. Algunos centros están asociados a bibliotecas universitarias, las cuales constituyen otros de los polos de desarrollo de las DH (por ejemplo, el eScholarship Research Centre en la University of Melbourne). Otro tipo de entidades responden mejor a la descripción de redes y asociaciones (18,6\%; por ejemplo, Hastac o el Linguistic Data Consortium), proyectos de investigación (7,1\%; Pegasus data project) o archivos digitales (6,4\%; Chaco Research Archive), entre otros.

Sólo 12 entidades $(7,7 \%)$ incluyen en su web contenidos bajo licencias Creative commons, como alternativa al copyright (por ejemplo: Ancient World Mapping Center; Margot, de la Univ. of Waterloo, Canadá; o ADHO).

Finalmente, sólo 20 entidades $(12,8 \%)$ contienen menciones expresas a la inclusión de las ciencias sociales dentro de su ámbito de actuación.

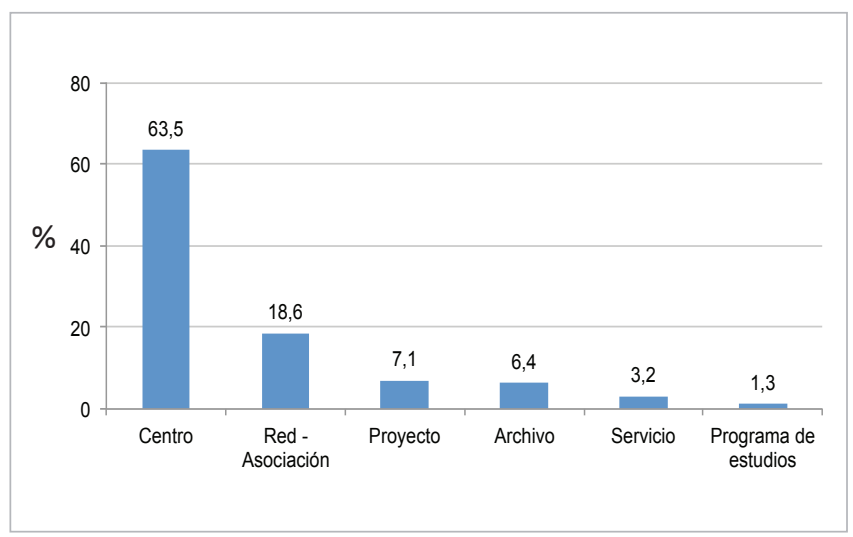

Gráfico 3. Centros de DH por tipología (en porcentaje) 


\subsection{Análisis de la naturaleza y objeto de los centros}

El análisis de los datos del proceso de codificación de centros dio lugar a los siguientes resultados (gráfico 4).

Con respecto a la categoría A sobre el propósito de los centros, los objetivos mayoritariamente destacados fueron: el fomento de la interdisciplinariedad (A5), el desarrollo de infraestructuras (A4) y el desarrollo de comunidades (A1). Tanto la crítica de formas tradicionales de conocimiento (A2) como la implicación social (A3) quedaron en un segundo plano.

El modo de alcanzar estos objetivos se centra principalmente en la generación de espacios físicos y virtuales para el encuentro de las comunidades interesadas (B2); el desarrollo de proyectos de investigación (B3); y la generación de infraestructuras (B1). En último lugar aparece la oferta de servicios de asesoramiento (B4).

Finalmente, tanto la reivindicación del papel de las humanidades en el contexto digital (C1) como el trabajo colaborativo e interdisciplinar (C2) constituyen los dos principios fundamentales que mueven su actividad. La apertura (C3), el compromiso social (C4) y lo que Zorich denominó el cuestionamiento de las "vacas sagradas" [carrera académica, necesidad de publicar, filtro del peer-review...] (C5), características más asociadas a la tradición de culturas digitales, presentan una menor relevancia.

Además de este análisis descriptivo, se han explorado posibles asociaciones entre las características de los centros y su presencia en redes sociales. Dado que la comunicación en redes sociales es propia del mundo digital, tomamos su grado de empleo por los centros como un indicador del grado de innovación y experimentación que diversas propuestas atribuyen a las DH (Digital humanities manifesto, 2009; Presner, 2012; McCarty, 2014). Sólo 78 centros cuentan con perfil en Twitter (50,0\%) y 48 en Facebook (30,8\%).

Los centros se clasificaron según su presencia en Twitter medida en función del número de tweets publicados, un indicador de la intención de generar interacciones sociales y de mantener presencia pública.

Se dividió la muestra en tres grupos: entidades sin presencia en Twitter (No_Twitter), con bajo uso (Bajo) y con alto

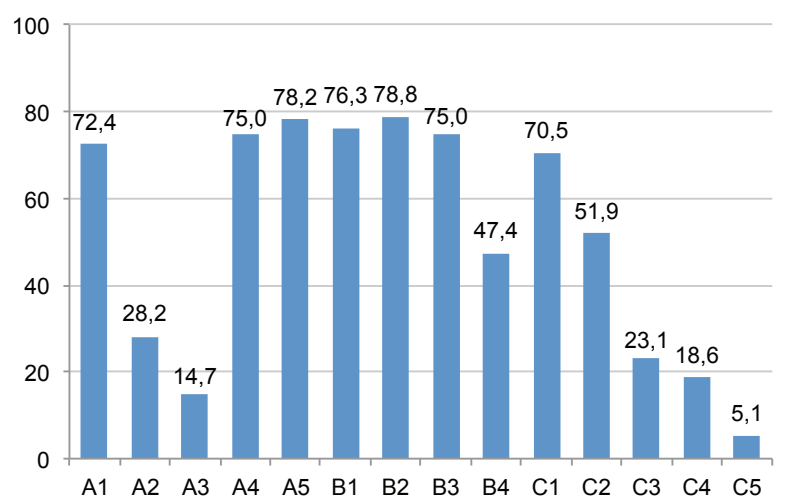

Gráfico 4. Frecuencias relativas de las características de los Centros de DH. uso (Alto). La distinción entre las categorías bajo y alto uso de Twitter se realizó tomando como referencia la media de tweets de todos los centros (media de publicaciones: 1.133,27; desviación típica 2.037,49).

Para cumplir con el objetivo anterior se realizó un análisis de correspondencias (correspondence analysis)(Greenacre, 2007) que permite examinar la asociación existente entre dos variables (presencia y uso de Twitter y naturaleza de los centros) a través de la tabla de contingencia que recoge las frecuencias entre ambas. El porcentaje de inercia de cada dimensión señala cuán importante es para la interpretación de los resultados. En la figura 5 el porcentaje de inercia del 90,90\% de la dimensión 1 indica que las distancias que han de ser tenidas en cuenta para la interpretación son las del eje horizontal. Así, se observa que las características A2 y C4 se encuentran muy asociadas, mientras que el grado de asociación de éstas con B1 o C4 es mucho menor.

El mapa de correspondencias muestra claramente cómo las características altamente asociadas con los centros con mayor presencia y uso de Twitter son aquellas que hacen referencia a una visión más comprometida de las $\mathrm{DH}$ desde el punto de vista de la crítica de las formas tradicionales de conocimiento y aprendizaje existentes y la formulación de propuestas innovadoras (A2), la responsabilidad cívica y social (C4) o el cuestionamiento del status quo (C5).

El resto de características se concentran en torno a los centros con un bajo uso de Twitter y sin Twitter. Estas características suelen presentar una frecuencia muy alta, lo cual indica que son rasgos que también pueden compartir los centros con una mayor presencia en redes; sin embargo, es conveniente notar que las asociaciones anteriormente indicadas (A2, C4, C5) corresponden a características con una frecuencia reducida lo que manifiesta la fuerza de dicha asociación.

Es particularmente significativo cómo las características vinculadas con las infraestructuras (A4, B1) se asocian con aquellos centros que no tienen presencia en Twitter, lo que se podría explicar por la coincidencia con el origen de muchos centros de $\mathrm{DH}$ como centros de provisión de infraestructuras tecnológicas en universidades. Como señala Svenson (2009), históricamente, incluso en nuestros días, se consideran los centros como unidades que proporcionan

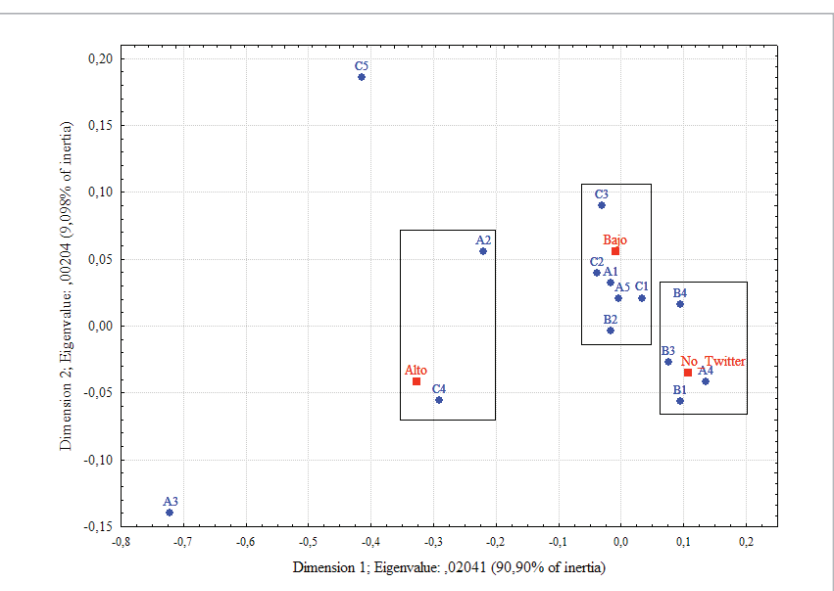

Gráfico 5. Asociación entre las características de los centros de $\mathrm{DH}$ y la presencia y uso de Twitter (mapa de correspondencias). 
servicios y funciones instrumentales a otras unidades universitarias. También es preciso destacar que aquellos centros orientados en su misión al desarrollo de comunidades para compartir recursos y experiencias (A1) y crear entornos de colaboración interdisciplinar (A5, B2) aparecen más asociados al grupo de Bajo uso pero en una posición más central entre los tres grupos.

\section{Discusión de resultados}

El análisis de los centros de DH incluidos en CenterNet pone en solfa algunas de los temas candentes en el campo. Los abordamos a continuación de manera sintética.

\section{Heterogeneidad en la organización de las DH}

Queda de manifiesto que existe una variada morfología en el modo en que se organizan las comunidades e iniciativas de DH. En primer lugar cabe señalar que el listado de "centros" que CenterNet recoge no incluye únicamente centros propiamente dichos (la mayoría), sino también redes, proyectos, programas de estudio y archivos. Sin duda cada uno de ellos compone una historia particular del modo en que las $\mathrm{DH}$ se han ido desarrollando en cada lugar. El papel de las redes es fundamental ya que el campo, por su carácter transversal pero a la vez especializado, se ha configurado de forma distribuida internacionalmente (si bien como veremos a continuación existe un fuerte sesgo anglosajón). Un ejemplo singular es Hastac (Humanities, Arts, Science, and Technology Alliance and Collaboratory), una red virtual con más de 10.000 miembros que trabaja en torno a grupos de trabajo online y organiza encuentros internacionales de forma periódica.

http://www.hastac.org

Igual sucede con las asociaciones, si bien en la muestra contamos con algunas no centradas exclusivamente en $\mathrm{DH}$ sino con alcance más general como por ejemplo la British Academy o el American Council of Learned Societies, en algunos casos desempeñando un papel relevante en la financiación y promoción de la investigación. Consideramos de gran interés que las DH encuentren acomodo dentro de estas organizaciones de amplio alcance ya que ello contribuye a su reconocimiento académico dentro de la amplia comunidad del conocimiento.

Sería conveniente en cualquier caso, solicitar a CenterNet, como red de centros de referencia, una mejor labor de curación de datos en su web, una clarificación de sus criterios para aceptar una entidad en su listado y una apertura hacia otros entornos culturales para evitar nuevas formas de coIonialismo en el campo.

\section{Anglocentrismo: nuevas formas de colonialismo}

Pese a que CenterNet es una iniciativa de la Alliance of Digital Humanities Organizations, es decir, tiene un enfoque claramente internacional, la imagen que cualquier lector desconocedor del panorama internacional de las $\mathrm{DH}$ podría llevarse a partir de su información es la de que la mayoría de las iniciativas se desarrollan en Estados Unidos o Reino Unido y en inglés. Existe una comunidad muy activa fuera de este ámbito, como es el caso del mundo hispano como bien dibuja Galina-Rusell (2013), donde la tradición viene de le- jos (Marcos-Marín, 1994; Rojas-Castro, 2013). La preocupación por una situación de colonialismo cultural en el campo se ha puesto de manifiesto por parte de Fiormonte (2012) y Dacos (2013), que apuntan al riesgo de exclusión de comunidades de investigadores y de las culturas sobre las que investigan.

Así, ningún centro del entorno hispano está representado en el retrato que formula CenterNet, echando en falta, entre otras, instituciones como la Asociación de Humanidades Digitales Hispánicas, la Red de Humanidades Digitales o la Red Aracne.

http://www.humanidadesdigitales.org http://www.humanidadesdigitales.net http://www.red-aracne.es

Creemos que es necesario seguir perseverando en iniciativas que den visibilidad a la comunidad de $\mathrm{DH}$ en español, por ejemplo, mediante el Día de humanidades digitales, que en 2013 promovió la Universidad Nacional Autónoma de México, como versión en español del Day of $D H$. http://dhd2013. filos.unam.mx

También mediante proyectos como el Atlas de humanidades y ciencias sociales digitales que pretende recopilar centros de investigación, proyectos, investigadores y recursos que se desarrollan en los ámbitos culturales del español y portugués. http://atlascshd.org

\section{¿Dónde situar los límites de las humanidades digita- les? Problemas de inclusión y exclusión}

La heterogeneidad mostrada en la variedad de formas de organización de los centros (con 6 tipos identificados como indica el gráfico 3), en el empleo de diversos idiomas (gráfico 2) o en la utilización de licencias para los contenidos publicados (copyright frente a licencias abiertas), se observa también en amplitud de disciplinas y temas que abordan los centros (desde los juegos, la investigación en lingüística, literatura, arte o museística). A lo largo de la última década se ha vivido un proceso paradójico según Rockwell (2011): humanities computing comienza como un campo minoritario y apartado de las humanidades en su conjunto. Sin embargo, la percepción actual por parte de investigadores con diversos intereses y adscripciones es que las $\mathrm{DH}$ son a su vez excluyentes. Así pues la consolidación del campo no está exenta de acalorados debates sobre problemas de inclusión y exclusión en el mismo (Svenson, 2009; Ramsay, 2011; Terras, 2011).

Una de las cuestiones que nos gustaría aportar a este debate es la inclusión o no de las ciencias sociales dentro del discurso (Rosenbloom, 2012; Spiro, 2014). El análisis muestra que aproximadamente el $13 \%$ de las organizaciones estudiadas las incluyen como parte de su misión. Es el caso de algunos centros como HyperStudio, iChass, o Crassh. En el caso de la International Association for Social Science Information Service and Technology (lassis) no se hace referencia alguna a las humanidades.

Si consideramos de forma amplia una idea de ciencias sociales y humanidades digitales, otros tipos de centros deberían tenerse también en cuenta (por ejemplo, Oxford Internet Institute, Berkman Center y centros de perfil similar). 


\section{Lo abierto y el compromiso social}

Ideas vinculadas al conocimiento abierto y a un mayor compromiso social aparecen recogidas en documentos como el Digital humanities manifesto (2009). El tema de lo abierto es significativo ya que forma parte de la cultura digital que se deriva del software libre y que alimenta buena parte de los debates sobre el digital scholarship (Weller, 2011). Sólo un 23\% de los centros hace alguna referencia explícita a esta cuestión, lo cual resulta escaso si, como apunta Russell (2014), lo abierto constituye un valor fundacional clave cuando pensamos en las redes en el siglo XXI. Algunos de los centros que abordan el tema son, entre otras, Matrix o el Hamburg Digital Humanities.

Adicionalmente, los centros cuyos contenidos en el sitio web se adhieren a licencias Creative commons, como indicador de su apoyo a la difusión abierta de conocimiento, son muy escasos $(7,7 \%)$, aunque esta situación podría verse afectada por las regulaciones de los países o de las universidades a las que pertenecen.

En relación con el compromiso social, es interesante explorar las relaciones entre public humanities y $\mathrm{DH}$ (es el caso del Digital Studio for Public Arts \& Humanities).

\section{Moderada presencia en medios sociales}

La presencia en redes sociales es moderada (Twitter, 50\%; Facebook, 30,8\%), especialmente si tenemos en cuenta las significativas diferencias en cuanto a nivel de publicaciones entre unos centros y otros. Sí ha quedado clara la vinculación con determinadas características de los centros, evidenciando como aún hay camino por recorrer en las humanidades digitales para asumir las ideas de las culturas digitales y del digital scholarship.

\section{Bibliografía}

Berry, David M. (ed.) (2012). Understanding digital humanities. Basingstoke: Palgrave, MacMillan. ISBN: 978 0230292659

Burdick, Anne; Drucker, Johanna; Lunenfeld, Peter et al. (2012). Digital humanities. MIT Press. ISBN: 9780262018470

Dacos, Marin (2013). "La stratégie du sauna finlandais". Blogo numericus.

http://bn.hypotheses.org/11138

Davidson, Cathy N. (2008). "Humanities 2.0: Promise, perils, predictions". PMLA, v. 123, n. 3, pp. 707-717.

http://dx.doi.org/10.1632/pmla.2008.123.3.707

Digital humanities manifesto (2009).

http://dev.cdh.ucla.edu/digitalhumanities/2008/12/15/ digital-humanities-manifesto

Fiormonte, Domenico (2014). "Humanidades digitales y diversidad cultural". Blog Infolet.

http://infolet.it/2014/02/01/humanidades-digitales-ydiversidad-cultural

Galina-Russell, Isabel (2013). "Is there anybody out there? Building a global digital humanities community". Blog humanidades digitales, 19 julio. Conferencia pronunciada en la clausura de la Digital humanities conference. http://goo.gl/9fEBkf
Gold, Matthew K. (ed.) (2012). Debates in the digital humanities. Minneapolis: University of Minnesota Press. ISBN: 9780816677955

Greenacre, Michael (2007). Correspondence analysis in practice. Boca Raton: Chapman \& Hall. ISBN: 9781584886167

Hockey, Susan (2000). Electronic texts in the humanities. Oxford: Oxford University Press. ISBN: 9780198711940

Kirschenbaum, Matthew G. (2010). "What is digital humanities and what's it doing in English departments?". Association of Departments of English (ADE) bulletin, n. 150.

http://mkirschenbaum. files.wordpress.com/2011/03/adefinal.pdf

Lucía-Megías, José-Manuel (2003). “La informática humanística: notas volanderas desde el ámbito hispánico". Incipit, n. 23, pp. 91-114.

Marcos-Marín, Francisco (1994). Informática y humanidades. Madrid: Gredos.

McCarty, Willard (2005). Humanities computing. Basingstoke and New York: Palgrave Macmillan. ISBN: 978 1403935045

McCarty, Willard (2014). "Getting there from here. Remembering the future of digital humanities. Roberto Busa award lecture 2013". Literary and linguist computing.

http://dx.doi.org/10.1093/Ilc/fqu022

Ortega, Élika; Gutiérrez, Silvia-Eunice (2014). “MapaDH. Una exploración de las humanidades digitales en español y portugués". En: Romero Frías, E. y Sánchez González, M. "Ciencias sociales y humanidades digitales. Técnicas, herramientas y experiencias de e-Research e investigación en colaboración". Cuadernos artesanos de comunicación.

http://mapahd.org/about

Presner, Todd (2012). "How to evaluate digital scholarship". Journal of digital humanities, v. 1, n. 4, Fall.

http://journalofdigitalhumanities.org/1-4/how-toevaluate-digital-scholarship-by-todd-presner

Ramsay, Stephen (2011). "On building". http://stephenramsay.us/text/2011/01/11/on-building

Rockwell, Geoffrey (2011). "Inclusion in the digital humanities". Philosophi.ca.

http://goo.gl/dZxEwW

Rojas-Castro, Antonio (2013). “El mapa y el territorio. Una aproximación histórico-bibliográfica a la emergencia de las humanidades digitales en España". Caracteres, v. 2, n. 2, nov. http://revistacaracteres.net/revista/vol2n2noviembre2013/ el-mapa-y-el-territorio

Rosenbloom, Paul S. (2012). "Toward a conceptual framework for the digital humanities". Digital humanities quarterly, v. 6, n. 2

http://www.digitalhumanities.org/dhq/vol/6/2/000127/000127. $h$ tml

Russell, Andrew L. (2014). Open standards and the digital age: History, ideology, and networks. Cambridge University Press. ISBN: 9781107612044 
Schreibman, Susan; Siemens, Ray; Unsworth, John M. (eds.) (2004). A companion to digital humanities. Oxford: Blackwell. http://www.digitalhumanities.org/companion

Siemens, Lynne (2009). "It's a team if you use "reply all": An exploration of research teams in digital humanities environments". Literary and linguistic computing, v. 24, n. 2, pp. 225233.

http://dx.doi.org/10.1093/llc/fap009

Spiro, Lisa (2014). "Defining digital social sciences". http://acrl.ala.org/dh/2014/04/09/defining-digital-socialsciences

Svensson, Patrik (2009). "Humanities computing as digital humanities". Digital humanities quarterly, v. 3, n. 3. http://digitalhumanities.org/dhq/vol/3/3/000065/000065.html

Terras, Melissa (2006). "Disciplined: Using educational studies to analyse "humanities computing'". Literary and linguistic computing, v. 21, n. 2, pp. 229-246.

http://dx.doi.org/10.1093/Ilc/fql022

Terras, Melissa (2011). "Peering inside the big tent: Digital humanities and the crisis of inclusion".

http://melissaterras.blogspot.co.uk/2011/07/peering-inside-

\section{big-tent-digital.html}

Terras, Melissa; Nyhan, Julianne; Vanhoutte, Edward (eds.) (2013). Defining digital humanities. A reader. Furnham, Surrey: Ashgate Publishing. ISBN: 9781409469636

Unsworth, John M. (2007). "Digital humanities centers as cyberinfrastructure". Digital humanities centers summit. Washington, DC, 12 April.

http://people.brandeis.edu/ unsworth/dhcs.html

Warwick, Claire (2004). "No such thing as humanities computing? An analytical history of digital resource creation and computing in the humanities". In: Association for Computers; Humanities-Association for Literary and Linguistic Computing Conference, University of Gothenburg, Sweden. http://eprints.ucl.ac.uk/155095

Weller, Martin (2011). The digital scholar. How technology is transforming scholarly practice. Bloomsbury Academic. ISBN: 9781849666176

Zorich, Diane M. (2008). "A survey of digital humanities centers in the United States". Washington, DC: Council on Library and Information Resources, 2008.

http://www.clir.org/pubs/abstract/pub143abst.html

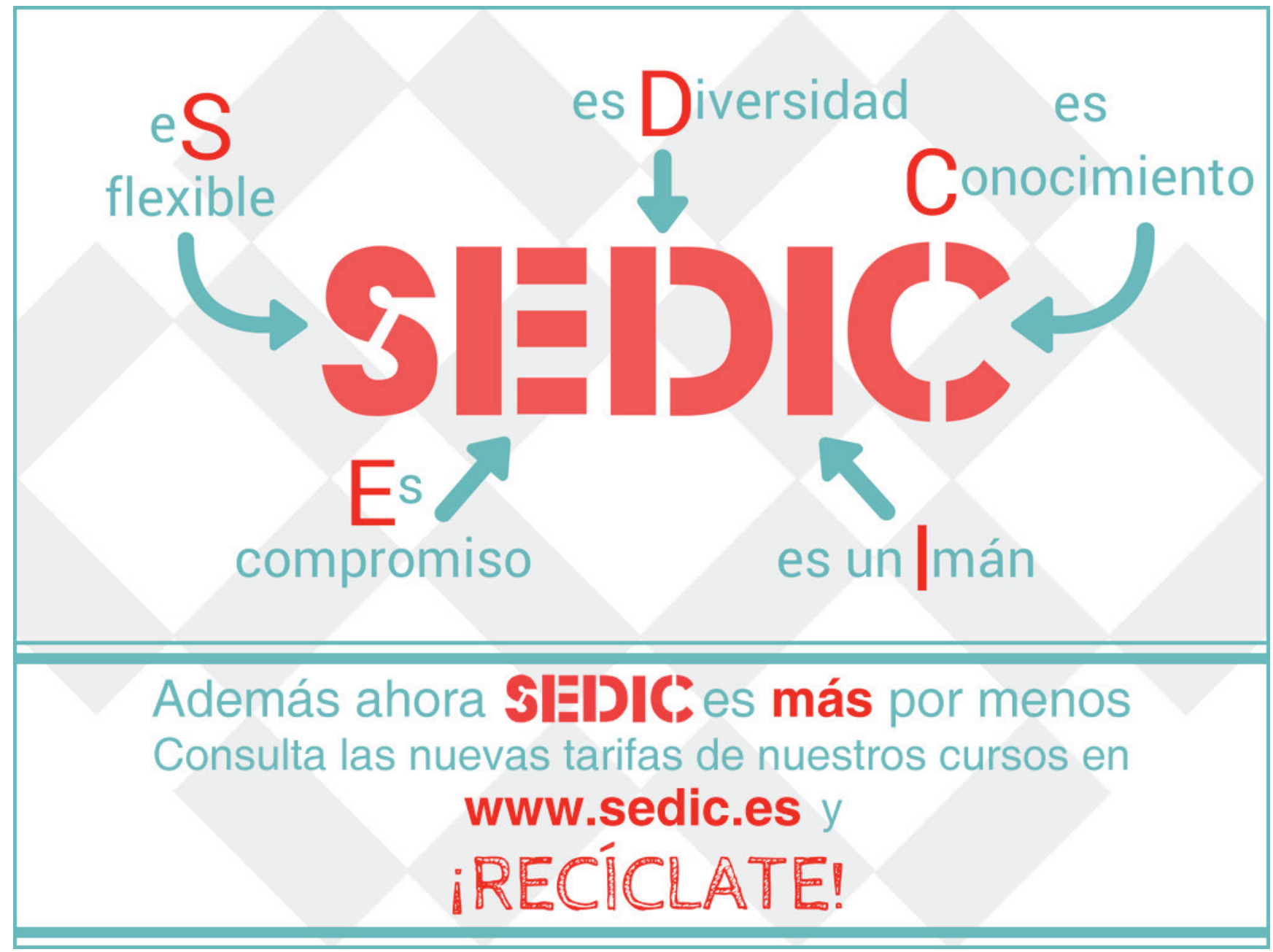

